\title{
A comparison of recombination frequencies in intraspecific versus interspecific mapping populations of Nasonia
}

\author{
LW Beukeboom ${ }^{1}$, O Niehuis ${ }^{2,3}$, BA Pannebakker ${ }^{1,4}$, T Koevoets ${ }^{1}$, JD Gibson², DM Shuker ${ }^{4,5}$, \\ $\mathrm{L}$ van de Zande $^{1}$ and $\mathrm{J}$ Gadau ${ }^{2}$ \\ ${ }^{1}$ Evolutionary Genetics, Centre for Ecological and Evolutionary Studies, University of Groningen, Haren, The Netherlands; ${ }^{2} S c h o o l ~ o f$ \\ Life Sciences, Arizona State University, Tempe, AZ, USA; ${ }^{3}$ Behavioural Biology, University of Osnabrück, Osnabrück, Germany and \\ ${ }^{4}$ Institute of Evolutionary Biology, School of Biological Sciences, University of Edinburgh, Edinburgh, UK
}

\begin{abstract}
We present the first intraspecific linkage map for Nasonia vitripennis based on molecular markers. The map consists of 36 new microsatellite markers, extracted from the Nasonia genome sequence, and spans $515 \mathrm{cM}$. The five inferred linkage groups correspond to the five chromosomes of Nasonia. Comparison of recombination frequencies of the marker intervals spread over the whole genome $(N=33$ marker intervals) between the intraspecific $N$. vitripennis map and an interspecific $N$. vitripennis $\times N$. giraulti map revealed a slightly higher $(1.8 \%)$ recombination frequency in the intraspecific cross. We further considered an $N$. vitripennis $\times N$. longicornis map with 29 microsatellite markers spanning $430 \mathrm{cM}$. Recom-
\end{abstract}

bination frequencies in the two interspecific crosses differed neither between reciprocal crosses nor between mapping populations of embryos and adults. No major chromosomal rearrangements were found for the analyzed genomic segments. The observed differential $F_{2}$ hybrid male mortality has no significant effect on the genome-wide recombination frequency in Nasonia. We conclude that interspecific crosses between the different Nasonia species, a hallmark of Nasonia genetics, are generally suitable for mapping quantitative and qualitative trait loci for species differences.

Heredity (2010) 104, 302-309; doi:10.1038/hdy.2009.185; published online 20 January 2010

Keywords: hybrids; linkage map; microsatellites; recombination; insects; speciation

\section{Introduction}

Genetic linkage maps are essential for the genetic analysis of biological traits. For example, research on the identification of the genetic architecture of quantitative traits relies heavily on the availability of linkage maps. Owing to the recent developments in DNA marker technologies, linkage maps are now available for many plant and animal species. Although this is true for many non-model as well as model organisms, the availability of the whole genome sequence for a model organism still greatly facilitates the rapid development of large numbers of reliable markers and, as a consequence, detailed linkage mapping using high throughput techniques. Traditionally, RAPD and AFLP markers were used in the absence of genome sequences because RAPD and AFLP genotyping was the only way to analyze a large number of variable markers necessary for linkage mapping. However, these markers are inherently difficult to interpret and are plagued with low reproducibility. The most commonly applied alternatives,

Correspondence: Dr LW Beukeboom, Centre for Ecological and Evolutionary Studies, University of Groningen, PO Box 14, Haren NL-9750 $A A$, The Netherlands.

E-mail: l.w.beukeboom@rug.nl

${ }^{5}$ Current address: School of Biology, University of St Andrews, St Andrews, KY16 9TH, UK

Received 7 May 2009; revised 16 October 2009; accepted 10

December 2009; published online 20 January 2010 microsatellites and single nucleotide polymorphism markers are more informative and robust, but their development typically requires a relatively large investment in terms of time and resources. The availability of genome sequence information avoids the costly cloning and sequencing efforts needed to detect markers, and one can detect markers and develop oligonucleotide primers for polymerase chain reaction (PCR) in silico. This approach allows for the rapid development of hundreds of microsatellite and single nucleotide polymorphism markers. An additional advantage of having the physical genome sequence of a study organism available is that one can use positional information to select markers with equal recombinational spacing across the genome (for example a marker every $20 \mathrm{cM}$ ), which greatly facilitates mapping of traits of interest. Moreover, one can compare local recombination frequencies with features of the physical map position to identify, for example, recombination hotspots, chromosomal inversions, and gene-rich genomic segments (Petes, 2001; Myers et al., 2005; Kulathinal et al., 2008; Niehuis et al., 2010; Werren et al., 2010).

Genetic linkage maps are now available for many insect species (Wilfert et al., 2007; Hunter and Kole, 2008). However, high-density maps are still restricted to species whose genomes have been sequenced: fruit flies of the genus Drosophila (Chen et al., 2009), the yellow fever mosquito Aedes aegypti (Fulton et al., 2001), the red flour beetle Tribolium castaneum (Lorenzen et al., 2005), the 
silkworm Bombyx mori (Yamamoto et al., 2006), and the honey bee Apis mellifera (Solignac et al., 2007). Here, we are concerned with the parasitoid (jewel) wasp Nasonia vitripennis, whose complete genome sequence has recently been published together with that of its closely related congeners $N$. giraulti and $N$. longicornis (Werren et al., 2010).

$N$. vitripennis has been used for genetic studies for over 60 years. Its suitability as an experimental organism stems largely from its ease of culturing in the laboratory and its haplodiploid mode of sex determination (Nasonia males are haploid). Saul (1993) published the first comprehensive linkage map for $N$. vitripennis, summarizing information of earlier mapping studies by, among others, Saul and Kayhart (1956) and Whiting (1967). This classical Nasonia map consists of 47 visible mutant markers in five linkage groups, consistent with the haploid complement of $N=5$ chromosomes. In recent years, Nasonia has become an important model organism in evolutionary, ecological, and developmental genetics, which culminated in the Nasonia genome project (Werren et al., 2010). The intense interest in this organism, in combination with the development of DNA marker technologies, has resulted in several efforts to construct molecular marker linkage maps for Nasonia (Gadau et al., 2008). The first linkage map using molecular markers for Nasonia stems from the pre-genome sequence era and was inferred using RAPD markers (Gadau et al., 1999). The first microsatellite loci of Nasonia were published by Pietsch et al. (2004), and, in the same year, Rütten et al. (2004) developed chromosome-specific microsatellite markers from microcloned chromosomes. This made it possible to homologize the earlier inferred linkage groups of Nasonia (Saul, 1993; Gadau et al., 1999) with its five chromosomes (Gokhman and Westendorf, 2000). Niehuis et al. (2008) then published an interspecific $N$. vitripennis $\times N$. giraulti map containing 38 molecular markers. The most recent linkage map for Nasonia is based on a large number of interspecific single nucleotide polymorphism markers identified from the annotated Nasonia genome sequences (1255 markers; Niehuis et al., 2010).

The genus Nasonia currently includes four species: $N$. vitripennis, $N$. longicornis, $N$. giraulti, and $N$. oneida; the latter being described in this issue (Raychoudhury et al., 2010). The divergence of $N$. vitripennis and the other three species is estimated to be around 1 mya, and that of $N$. longicornis and $N$. giraulti about 0.2 mya (Campbell et al., 1993). The Nasonia species complex has become especially instructive for genetic analysis of species differences, because all the species can be crossed and genomic segments easily exchanged between species. As such, a number of studies have used linkage maps constructed from interspecific crosses. However, an intraspecific Nasonia map based on molecular markers has yet to be published. Importantly, linkage maps based on interspecific crosses may yield incorrect recombination distance estimates between markers or genes of interest because of hybridization artifacts. Specifically, recombination frequencies may be increased or decreased when combining genomes of two species because of major genome reorganizations or the release of recombination suppressors (Trickett and Butlin, 1994; Zhang et al., 1999). Moreover, the recovery of alleles may be affected by nuclear-nuclear and nuclear-cytoplasmic genic incom- patibilities, both of which are known to occur in Nasonia hybrids (Breeuwer and Werren, 1995; Gadau et al., 1999; Niehuis et al., 2008). Weston et al. (1999) have presented a formal model, which suggested that hybrid mortality because of nucleo-nucleo genic incompatibilities can lead to pseudo-linkage and thus the inference of a false marker order. To test for marker transmission ratio distortion (MTRD) in Nasonia hybrids, Niehuis et al. (2008) compared marker allele frequencies between embryos and adults. They found that some markers indeed show a significantly distorted recovery in adults compared with embryos and attributed the MTRD to mortality associated with particular genotypes during larval development. However, to what extent MTRD in hybrids impacts on the accuracy of interspecific linkage maps remains to be tested.

Here, we present the first intraspecific linkage map for $N$. vitripennis based on molecular markers. Considering the chromosome-anchored markers developed by Rütten et al. (2004), we were able to associate the original linkage groups of N. vitripennis distinguished by Saul (1993) with the five Nasonia chromosomes. We contrast recombination frequencies of homologous genomic segments between the intraspecific $N$. vitripennis cross and an interspecific $N$. vitripennis $\times N$. giraulti mapping population of embryos that showed no MTRD using the data published by Niehuis et al. (2010). We next compare recombination frequencies of embryos with those of adults in two interspecific crosses (that is $N$. vitripennis $\times N$. longicornis and $N$. vitripennis $\times N$. giraulti) to determine the effect of MTRD on recombination estimates and marker order. Finally, we test for differences in recombination frequencies between both reciprocal crosses of the two interspecific mapping populations to screen for non-recombining genome segments.

\section{Materials and methods}

\section{Microsatellite marker selection}

We used the position and properties of microsatellite markers from an in silico screen of the $N$. vitripennis genome sequences (Werren et al., 2010) conducted by Pannebakker et al. (2010). We selected microsatellite markers primarily on the largest $N$. vitripennis scaffolds and looked for flanking sequences with ortholog sequences in the $N$. giraulti and $N$. longicornis trace sequence archives. We initially selected two microsatellite markers per scaffold, one on either end, to be able to map and orient the scaffolds along the chromosomes. Although the microsatellite markers of the Nv100 series (see Supplementary Table 1) were specifically screened for polymorphisms within $N$. vitripennis, those of the Nv300 series were tailored to match and/or replace molecular markers that had been used by Niehuis et al. (2008) to infer an $N$. giraulti $\times N$. vitripennis framework map.

\section{Mapping populations and marker genotyping}

All earlier mapping efforts in Nasonia exploited the advantage of haploid males. Typically, two parental lines differing in allele composition were crossed. The resulting $F_{1}$ females layed eggs as virgins, yielding recombinant $F_{2}$ males that carry either one of the grandparental alleles. 
Intraspecific $N$. vitripennis map: An initial screen of a number of different field strains for genetic variation (Pannebakker et al., 2008) provided two strains that showed allelic differences in a large number of loci: C222a (collected in 2003 by LW Beukeboom) and HV6 (collected in 2004 by T Koevoets, MN Burton-Chellew, and EM Sykes; for collection details see Burton-Chellew et al. (2008)). Both strains originated from De Hoge Veluwe in the Netherlands and were set up and maintained as iso-female lines (that is started as a line from a single mated female). A total of $276 \mathrm{~F}_{2}$ adult males of a cross between a C222a male and a HV6 female were genotyped for 41 microsatellite markers that are polymorphic between these two strains (36 of these markers could be mapped). DNA was extracted using 5\% Chelex 100 solution (Bio-Rad, Hercules, CA, USA). PCR amplifications of microsatellite markers of the Nv100 series were performed in $10.0 \mu \mathrm{l}$ volumes $\left(1 \times \mathrm{NH}_{4}\right.$ buffer, $10 \mathrm{mM} \mathrm{MgCl}$, $1 \mathrm{mM}$ dNTP mix, $4 \mathrm{mM}$ of each primer, 0.5 units BIOTAQ DNA polymerase (Bioline Ltd., London, UK), $2 \mu$ l DNA (20 ng)). The PCR temperature profile started with a 4 min denaturation step at $94{ }^{\circ} \mathrm{C}$ followed by 39 cycles of $30 \mathrm{~s}$ at $94^{\circ} \mathrm{C}, 30 \mathrm{~s}$ at the annealing temperature $\left(T_{\mathrm{a}}\right)$ specified in Supplementary Table 1, and $1 \mathrm{~min}$ at $72{ }^{\circ} \mathrm{C}$, followed by $10 \mathrm{~min}$ at $72{ }^{\circ} \mathrm{C}$. Differences in the length of the PCR products were determined on $4 \%$ Tris-acetate-EDTA agarose gels stained with ethidium bromide. Gels were run on a Sub-Cell Model 96 electrophoresis cell (Bio-Rad) at $120 \mathrm{~V}$ for a length of $\sim 5 \mathrm{~cm}$.

Interspecific $N$. vitripennis $\times N$. giraulti map: We analyzed genotype data of 120 embryonic and of 120 adult $F_{2}$ hybrid males of reciprocal crosses between $N$. vitripennis (AsymCX) and N. giraulti (RV2X) (see Niehuis et al., 2008, for methodological details). This allowed for a comparison of the recombination frequencies between embryos and adults within crosses and of embryos and adults between reciprocal crosses. All individuals had been genotyped for 38 single nucleotide polymorphism markers by Niehuis et al. (2008).

Interspecific $N$. vitripennis $\times N$. longicornis map: In a similar manner to the $N$. vitripennis $\times N$. giraulti interspecific cross, we genotyped 120 embryonic and 120 adult $F_{2}$ hybrid males of each reciprocal cross between $N$. vitripennis (AsymCX) and $N$. longicornis $(\mathrm{IV7}(\mathrm{U}))$. However, this time we used microsatellite markers that had been developed to match the molecular markers used by Niehuis et al. (2008). PCR amplification of the microsatellite markers of the Nv300 was conducted with the Qiagen multiplex PCR kit for five different sets according to the manufacturer's recommendations and applying Applied Biosystems Veriti and Applied Biosystems 9700 thermocyclers. The PCR temperature profile started with a $15 \mathrm{~min}$ denaturation step at $95^{\circ} \mathrm{C}$ followed by 30 cycles of $30 \mathrm{~s}$ at $94{ }^{\circ} \mathrm{C}, 1 \mathrm{~min} 30 \mathrm{~s}$ at the annealing temperature $\left(T_{\mathrm{a}}\right)$ specified in Supplementary Table 1 , and $1 \mathrm{~min}$ at $72{ }^{\circ} \mathrm{C}$, followed by $45 \mathrm{~min}$ at $72{ }^{\circ} \mathrm{C}$. All amplicons of the Nv300 microsatellite marker series were separated on an Applied Biosystems 3730 DNA Analyzer and analyzed with the software GeneMapper v4.0 (Applied Biosystems, Foster City, CA, USA).
Marker segregation data were analyzed with the software Multipoint (http://multiqtl.com), which infers marker order and map distances using a multipoint likelihood approach (Mester et al., 2003a,b, 2004). We calculated pairwise recombination fractions for all pairs of markers using the Kosambi mapping function (Kosambi, 1944).

\section{Comparison of recombination frequencies}

We compared recombination frequencies only between adjacent markers that were shared by the two respective populations. If a marker was present in only one of the two compared mapping populations, we excluded it from the analysis. In some cases, markers could not be unambiguously placed on the map. However, it was still possible to use them to estimate pairwise recombination frequencies (see Table 1). In some instances, the two maps differed in marker order, in which case the STS map of Niehuis et al. (2010) was used as template.

Intraspecific $N$. vitripennis versus interspecific $N$. vitripennis $\times N$. giraulti $\mathrm{F}_{2}$ hybrid embryos: To match the microsatellite markers used in the $N$. vitripennis intraspecific cross with the molecular markers that had been analyzed in the interspecific $N$. giraulti $(\hat{\delta}) \times N$. vitripennis ( $q$ ) cross, we searched for molecular markers in the interspecific map that were physically closest to the newly developed microsatellite markers (Nv100 series). Of the 38 microsatellite loci on the intraspecific map that we matched to the molecular markers on the interspecific map (Niehuis et al., 2008), one was identical (Nv319) and the remaining 37 showed an average distance of $0.2 \%$ recombination (range 0-2.7\% recombination, see Supplementary Table 2) based on the high-density linkage map published by Niehuis et al. (2010).

Embryo versus adult $\mathrm{F}_{2}$ hybrids: As a subset of markers were similarly genotyped in embryo and adult $N$. vitripennis $\times N$. giraulti $\mathrm{F}_{2}$ hybrid males, as well as in $N$. vitripennis $\times N$. longicornis $\mathrm{F}_{2}$ hybrid males, we were able to compare recombination frequencies between marker pairs directly.

Interspecific $N$. vitripennis $\times N$. giraulti versus interspecific $N$. vitripennis $\times N$. longicornis $F_{2}$ hybrids: To compare recombination frequencies between the $N$. vitripennis $\times$ $N$. giraulti and $N$. vitripennis $\times N$. longicornis interspecific crosses, molecular markers studied by Niehuis et al. (2008) were again matched for location with existing microsatellite markers (mostly Nv300 series). Of those matches, one was identical (Nv26) and 16 were on average separated by $1.0 \%$ recombination (range $0-5.4 \%$ recombination, see Supplementary Table 3).

\section{Results}

\section{Linkage maps}

In total, we developed 53 new dinucleotide and 3 trinucleotide microsatellite markers (together comprising the Nv100 and Nv300 marker series) from the Nasonia genome assembly 1.0 (Supplementary Table 1). For many of those loci, the three Nasonia strains sequenced in the Nasonia genome sequencing project had fixed allelic differences, and 35 of these new markers (plus one old 
Table 1 Markers and recombination frequency estimates used in the comparison between the intraspecific mapping population of adult male $N$. vitripennis and the interspecific mapping population of $N$. giraulti $\left(\delta^{\dagger}\right) \times N$. vitripennis $(q) F_{2}$ hybrid embryos (see Figure 2 )

\begin{tabular}{|c|c|c|c|c|}
\hline Chromosome & $\begin{array}{l}\text { Adjacent microsatellite } \\
\text { marker pair of } \\
\text { intraspecific } N \text {. } \\
\text { vitripennis map }\end{array}$ & $\begin{array}{l}\text { Recombination } \\
\text { rate estimate } \\
\text { in } N \text {. vitripennis } \\
\text { adults (cM) }\end{array}$ & $\begin{array}{l}\text { Corresponding adjacent scaffold positions } \\
\text { in N. vitripennis genome }\end{array}$ & $\begin{array}{l}\text { Recombination } \\
\text { rate estimate in } \\
\text { N. vitripennis- } \\
\text { N. giraulti } \\
\text { embryos (cM) }\end{array}$ \\
\hline 1 & Nv180-Nv181 & 0.145 & NVG SCAFFOLD16 1815958-NVG SCAFFOLD16 3425262 & 0.143 \\
\hline 1 & Nv181-Nv126 ${ }^{a}$ & 0.197 & NVG_SCAFFOLD16_3425262-NVG_SCAFFOLD41_300676 & 0.181 \\
\hline 1 & Nv126-Nv156 ${ }^{a}$ & 0.000 & NVG SCAFFOLD41 300676-NVG SCAFFOLD73 410408 & 0.000 \\
\hline 1 & Nv156-Nv170 $0^{a}$ & 0.000 & NVG SCAFFOLD73 410408-NVG SCAFFOLD170 1506160 & 0.008 \\
\hline 1 & Nv170-Nv191 ${ }^{\mathrm{a}}$ & 0.045 & NVG_SCAFFOLD170_1506160-NVG_SCAFFOLD33_767863 & 0.000 \\
\hline 1 & Nv191-Nv169 ${ }^{a}$ & 0.000 & NVG SCAFFOLD33 767863-NVG SCAFFOLD25 803967 & 0.000 \\
\hline 1 & Nv169-Nv105 & 0.136 & NVG_SCAFFOLD25_803967-NVG_SCAFFOLD1_2289896 & 0.039 \\
\hline 1 & Nv105-Nv127 & 0.221 & NVG_SCAFFOLD1_2289896-NVG_SCAFFOLD60_741110 & 0.136 \\
\hline 2 & Nv193-Nv20 & 0.352 & NVG_SCAFFOLD8_907155-NVG_SCAFFOLD31_231950 & 0.274 \\
\hline 2 & Nv20-Nv168 & 0.063 & NVG_SCAFFOLD31_231950-NVG_SCAFFOLD15_2695394 & 0.159 \\
\hline 2 & Nv168-Nv123 & 0.027 & NVG_SCAFFOLD15_2695394-NVG_SCAFFOLD13̄_2593414 & 0.023 \\
\hline 2 & Nv123-Nv132 & 0.012 & NVG_SCAFFOLD13_2593414-NVG_SCAFFOLD19_473227 & 0.012 \\
\hline 2 & Nv132-Nv133 & 0.071 & NVG SCAFFOLD19 473227-NVG SCAFFOLD19 2929250 & 0.063 \\
\hline 2 & Nv133-Nv186 & 0.211 & NVG_SCAFFOLD19_2929250-NVG__SCAFFOLD24̄_1685756 & 0.199 \\
\hline 2 & Nv186-Nv190 & 0.214 & NVG_SCAFFOLD24_1685756-NVG_SCAFFOLD309_48837 & 0.213 \\
\hline 3 & Nv319-Nv192 & 0.264 & NVG_SCAFFOLD18_2635495-NVG_SCAFFOLD6_1855275 & 0.271 \\
\hline 3 & Nv192-Nv189 & 0.054 & NVG SCAFFOLD6 $1855275-N V G$ SCAFFOLD44 4553601 & 0.071 \\
\hline 3 & Nv189-Nv184 & 0.072 & NVG_SCAFFOLD44__4553601-NVG__SCAFFOLD22__1311250 & 0.056 \\
\hline 3 & Nv184-Nv111 & 0.151 & NVG_SCAFFOLD22_1311250-NVG_SCAFFOLD17_1342149 & 0.099 \\
\hline 3 & Nv111-Nv108 & 0.175 & NVG_SCAFFOLD17_1342149-NVG_SCAFFOLD28_187258 & 0.177 \\
\hline 3 & Nv108-Nv107 & 0.139 & NVG_SCAFFOLD28_187258-NVG_SCAFFOLD28_1356359 & 0.141 \\
\hline 4 & Nv26-Nv141 & 0.378 & NVG_SCAFFOLD4_2791862-NVG_SCAFFOLD29_1439582 & 0.285 \\
\hline 4 & Nv141-Nv114 & 0.027 & NVG_SCAFFOLD29_1439582-NVG_SCAFFOLD23_1489254 & 0.054 \\
\hline 4 & Nv114-Nv147 & 0.027 & NVG_SCAFFOLD23_1489254-NVG_SCAFFOLD34_1272544 & 0.008 \\
\hline 4 & Nv147-Nv136 ${ }^{\text {a }}$ & 0.018 & NVG_SCAFFOLD34_1272544-NVG_SCAFFOLD26_7306 & 0.011 \\
\hline 4 & Nv136-Nv137 ${ }^{\mathrm{a}}$ & 0.004 & NVG_SCAFFOLD26_7306-NVG_SCAFFOLD26_1516282 & 0.004 \\
\hline 4 & Nv137-Nv182 & 0.090 & NVG_SCAFFOLD26_1516282-NVG_SCAFFOLD123_174844 & 0.065 \\
\hline 4 & Nv182-Nv154 & 0.202 & NVG_SCAFFOLD123_174844-NVG_SCAFFOLD9_3107879 & 0.162 \\
\hline 4 & Nv154-Nv104 & 0.329 & NVG_SCAFFOLD9_3107879-NVG_SCAFFOLD9_635808 & 0.318 \\
\hline 5 & Nv124-Nv179 & 0.291 & NVG_SCAFFOLD14_227534-NVG_SCAFFOLD14_3478710 & 0.287 \\
\hline 5 & Nv179-Nv152 & 0.105 & NVG_SCAFFOLD14_3478710-NVG_SCAFFOLD38_42794 & 0.038 \\
\hline 5 & Nv152-Nv176 & 0.185 & NVG_SCAFFOLD38_42794-NVG_SCAFFOLD1_6038985 & 0.127 \\
\hline 5 & Nv176-Nv109 & 0.008 & NVG_SCAFFOLD1_6038985-NVG__SCAFFOLD'̄1_7302524 & 0.007 \\
\hline
\end{tabular}

The recombination rate estimates are based on the number of recombinant individuals for each pair of adjacent markers shared in both crosses. Deviations in marker composition and position from the map in Figure 1 are due to the non-mapping of certain markers.

${ }^{a}$ Incongruent marker sequence: the scaffold positions are taken as template.

${ }^{\mathrm{b}}$ Not in intraspecific $N$. vitripennis map.

marker, Nv26) could be mapped using the intraspecific N. vitripennis mapping population.

Intraspecific $N$. vitripennis map: The map is based on a cross between two N. vitripennis field strains (C222a and HV6) and has a size of $515 \mathrm{cM}$. The studied microsatellite markers are distributed over all five linkage groups, representing the five chromosomes. The average marker spacing is $10.0 \mathrm{cM}$ (Figure 1).

Interspecific $N$. vitripennis $\times N$. longicornis map: The microsatellite markers used to infer the map were closely linked to the molecular markers studied by Niehuis et al. (2008). The map consists of 29 microsatellite markers in five linkage groups and spans $438 \mathrm{cM}$ (the map will be published elsewhere).

\section{Comparison of recombination frequencies}

Intraspecific versus interspecific recombination frequencies: We compared the recombination frequencies of 33 genome segments distributed over the entire linkage map for adult $N$. vitripennis males (map size $515 \mathrm{cM}$, average segment size $12.8 \mathrm{cM}$ ) and interspecific $N$. vitripennis $\times N$. giraulti embryos with giraulti cytoplasm (map size $381.5 \mathrm{cM}$, average segment size 11.0 cM; Niehuis et al., 2008; see Table 1). The 33 marker intervals span a total of $313.5 \mathrm{cM}$, which is about $70 \%$ of the saturated high-density genome map (Niehuis et al., 2010). We chose to use embryos of the interspecific cross to eliminate the possible effect of hybrid incompatibilities on recombination, potentially allowing us to identify genome reorganizations. Embryos of this particular cross do not show significant MTRD (Niehuis et al., 2008). Pairwise comparison of recombination frequencies between the intraspecific and the interspecific map revealed a slightly higher (1.8\%) intraspecific recombination rate on a genome-wide level $(N=33$ genomic segments, Wilcoxon matchedpairs test, $P=0.005$; Figure 2). Chromosome 1 and 5 show the largest deviations. This partly results from 


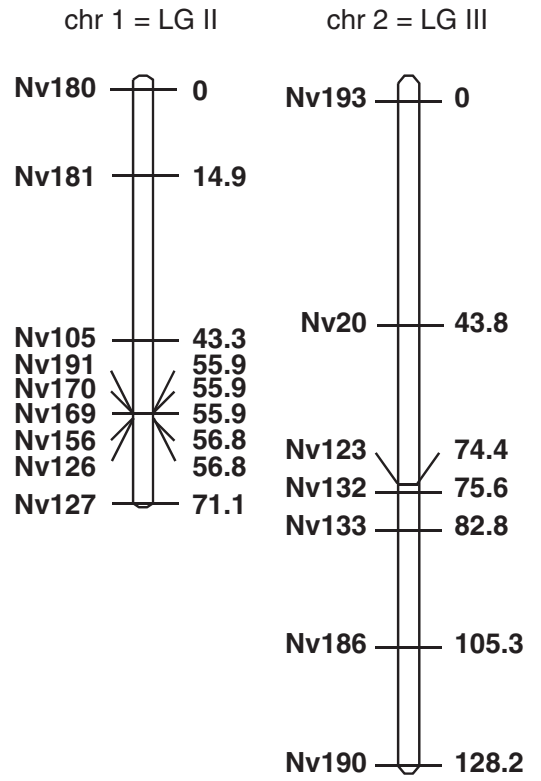

$\operatorname{chr} 3=$ LG V

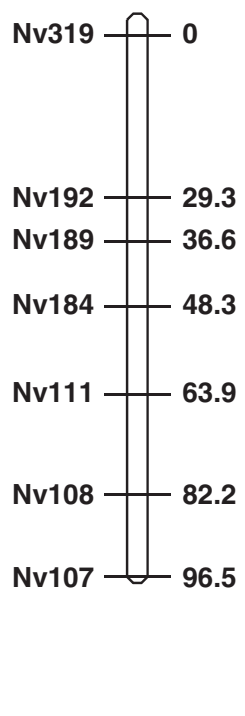

$\operatorname{chr} 4=$ LG IV

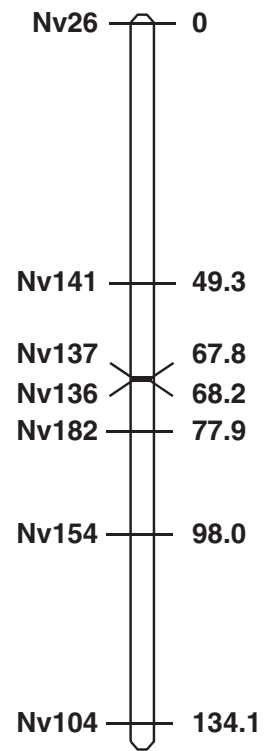

$\operatorname{chr} 5=$ LG I

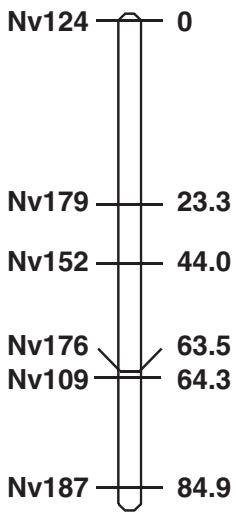

Figure 1 Genetic linkage map for $N$. vitripennis. The map comprises 36 microsatellite markers on five linkage groups, spanning a total of 515 cM. Saul's (1993) linkage groups were linked with the chromosome numbers by Rütten et al. (2004). Markers are shown on the left-hand side of each chromosome and map distances (in cM; Kosambi, 1944) are given on the right.

incongruity in the marker order between the two maps (in particular, the $28 \mathrm{cM}$ interval between the markers Nv105 and Nv127 on chromosome 1; Figure 1). When we removed all markers with inconsistent positions of chromosome 1, the average difference in the recombination frequency of markers on this chromosome shrinked from $2.9 \% \quad(N=8$ genome segments) to $0.2 \% \quad(N=1)$, and the genome-wide difference in recombination rate was $1.3 \%(N=26)$.

Embryonic versus adult $F_{2}$ hybrids: We were able to compare the recombination frequencies between embryos and adults of both reciprocal $N$. vitripennis $\times N$. giraulti crosses for 34 genomic segments (Figure 3). Genome-wide differences in recombination frequencies of embryos and adults were small and not significant in either cross (absolute difference was $2.9 \%$ for the $N$. giraulti $\left(\delta^{*}\right) \times$ $N$. vitripennis (q) cross and $2.4 \%$ for the $N$. vitripennis $($ đ) $\times N$. giraulti ( $q$ ) cross, $N=34$ genomic segments, Wilcoxon matched-pairs test, $P>0.05$; Figure 3 ).

The comparison of recombination frequencies between embryos and adults in the interspecific $N$. vitripennis $\times$ N. longicornis cross yielded comparable results (Figure 3 ). No significant genome-wide differences in recombination between embryos and adults were found in either cross (absolute difference between adults and embryos were $3.8 \%$ for hybrids with $N$. vitripennis cytoplasm and $3.9 \%$ for those with $N$. longicornis cytoplasm; $N=24$ genomic segments, Wilcoxon matched-pairs test, $P>0.05$ ). Recombination frequencies of adults versus adults and embryos versus embryos did not differ significantly between the reciprocal crosses of both species combinations either (Wilcoxon matched-pairs test, $P>0.05$ ).

Interspecific $N$. vitripennis $\times N$. giraulti versus interspecific $N$. vitripennis $\times N$. longicornis $F_{2}$ hybrids: Comparing $F_{2}$ hybrids with identical cytoplasm between interspecific

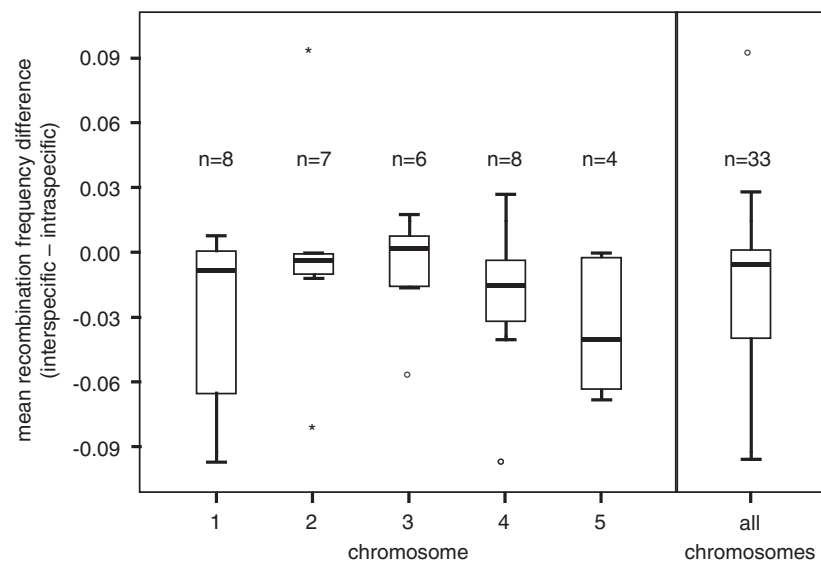

Figure 2 Comparison of recombination frequencies between $N$. vitripennis adult males and interspecific $N$. giraulti $\left(\delta^{\star}\right) \times$ $N$. vitripennis ( $(+)$ embryos. The left box plot shows differences in the recombination frequency between homologous genome segments per chromosome. The right plot depicts the differences pooled for the whole genome. In both cases, negative values indicate greater intraspecific recombination. Box plots show the median (thick horizontal line within the box), the 25 and 75 percentiles (box), and 1.5 times the interquartile range of the data (thin horizontal lines). Outliers are indicated by an open circle and extreme cases are marked by an asterisk.

$N$. vitripennis $\times N$. giraulti and $N$. vitripennis $\times N$. longicornis crosses did not indicate any significant differences in recombination rates for both adults and embryos $(N=12$ genomic segments; Wilcoxon matchedpairs test, $P>0.05$; Figure 4). The 12 marker intervals span a total of $199 \mathrm{cM}, \sim 45 \%$ of the saturated highdensity map (Niehuis et al., 2010). No matching intervals were available on chromosome 5, however. Hybrids 


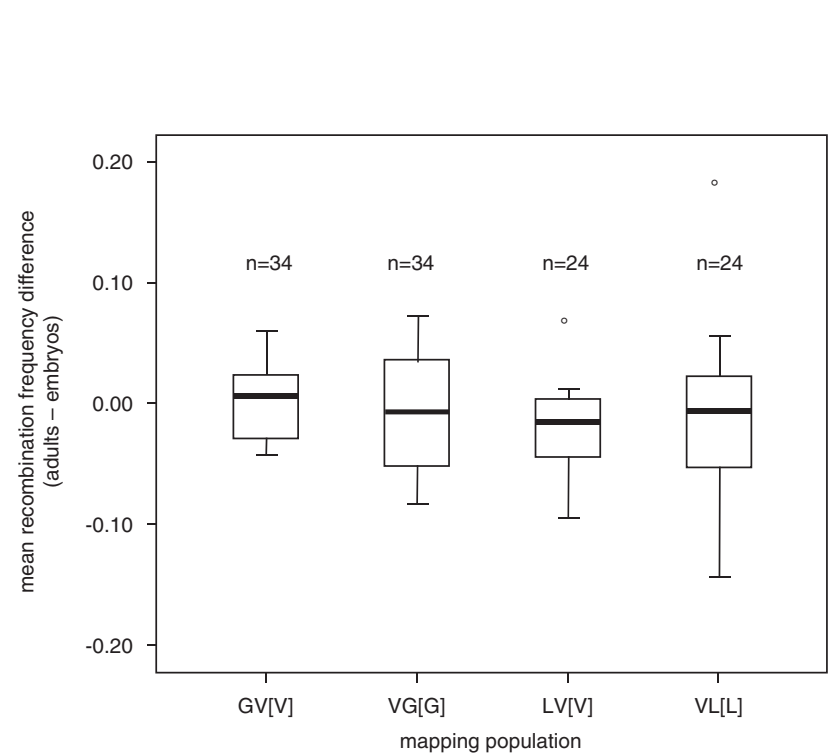

Figure 3 Comparison of recombination frequencies between mapping populations of $F_{2}$ hybrid embryos and adults from four interspecific crosses ( $N$. vitripennis $\times N$. giraulti and $N$. vitripennis $\times$ $N$. longicornis and the two respective reciprocal crosses). The nuclear genome of the parental species is indicated by a two-letter code $(\mathrm{V}=N$. vitripennis, $\mathrm{G}=N$. giraulti, $\mathrm{L}=N$. longicornis $)$. The genotype of the cytoplasm is shown in brackets (for example [V]= $N$. vitripennis cytoplasm). Negative values indicate greater recombination in embryos. Box plots show the median (thick horizontal line within the box), the 25 and 75 percentiles (box), and 1.5 times the interquartile range (thin horizontal lines) of the differences between homologous genome segments defined by flanking shared markers. Outliers are indicated by an open circle.

between $N$. vitripennis and $N$. giraulti and $N$. longicornis, respectively, did not show significant differences in recombination rates either $(N=12$ genomic segments; Wilcoxon matched-pairs test, $P>0.05$; Figure 4$)$.

\section{Discussion}

The first genetic linkage map of Nasonia based on RAPD markers had been inferred by analyzing an interspecific cross between N. vitripennis and N. giraulti (Gadau et al., 1999). RAPD and AFLP markers typically show higher rates of deviation from expected Mendelian segregation ratios than microsatellite markers (Voorrips et al., 1997; Tan et al., 2001; Zhang et al., 2007), likely because of genotyping errors. We found the same to be true for Nasonia (RAPD: C Pietsch, unpublished; AFLP: LW Beukeboom, unpublished). The first microsatellite markers for Nasonia were developed by Pietsch et al. (2004) and Rütten et al. (2004). The recently published genome sequences of three Nasonia species made it possible to rapidly develop additional microsatellite markers with both intraspecific variability and species-specific alleles (Pannebakker et al., 2010). These newly developed markers allowed us to quickly construct the first intraspecific linkage map for $N$. vitripennis based on molecular markers and to infer additional interspecific maps as well (that is $N$. vitripennis $\times N$. longicornis).

One potential problem with genotyping interspecific hybrids for genome mapping are deleterious interactions between the two genomes that can result in MTRD, in particular, in haploid males in which recessive deleterious interactions are not masked. Crosses between Nasonia species are known to result in nucleo-nucleo and nucleo-cytoplasmic ( = cytonuclear) genic incompat-

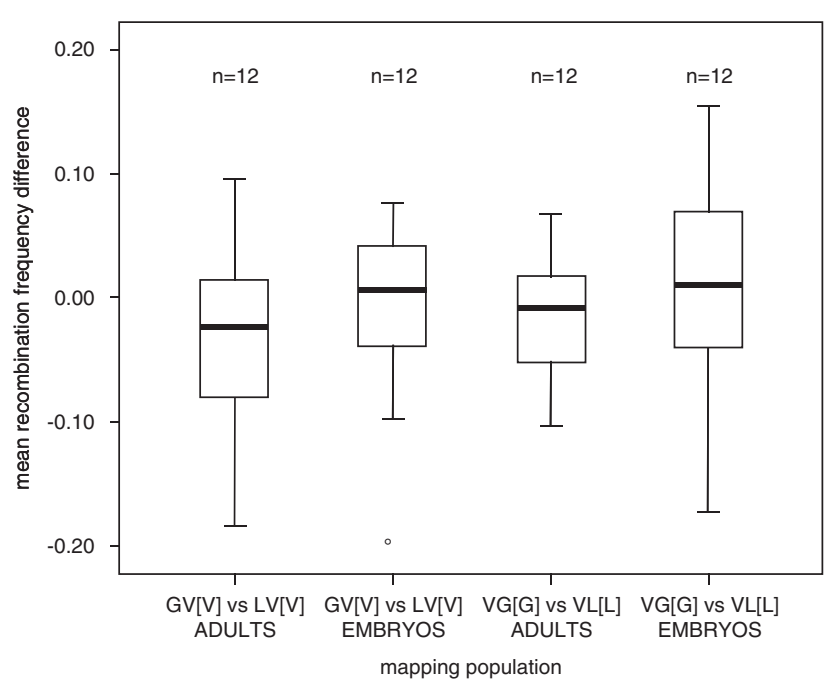

Figure 4 Comparison of the genome-wide recombination frequencies between eight independent interspecific mapping populations: adults and embryos of both reciprocal $N$. vitripennis $\times N$. giraulti and $N$. vitripennis $\times N$. longicornis crosses. The mean recombination frequency differences were not significantly different between the interspecific comparisons, irrespective of whether embryos or adults had been studied. The nuclear genome of the parental species is indicated by a two-letter code $(\mathrm{V}=N$. vitripennis, $\mathrm{G}=$ $N$. giraulti, $\mathrm{L}=N$. longicornis). Negative values indicate greater recombination in the mapping population given second in the comparison. The origin of the cytoplasm is shown in brackets (for example $[\mathrm{V}]=N$. vitripennis cytoplasm). Box plots show the median (thick horizontal line within the box), the 25 and 75 percentiles (box), and 1.5 times the interquartile range (thin horizontal lines) of the data. Outliers are indicated by an open circle.

ibilities that cause an increase in hybrid mortality during larval development (Breeuwer and Werren, 1995; Gadau et al., 1999; Niehuis et al., 2008). A major concern in Nasonia research on speciation and species differences when studying interspecific hybrids has therefore been whether interspecific linkage maps accurately reflect the order and distance between markers. This concern led Niehuis et al. (2008) to compare allele recovery rates between embryos and adults of interspecific hybrids. On four of the five chromosomes, they found at least one region at which markers were significantly distorted. Using the same samples as these authors, we found, however, that the presence of such distorters do not significantly affect the observed genome-wide recombination frequencies, as the linkage maps based on embryos or adults have similar sizes (Figure 2). Since we compared recombination frequencies between adjacent markers that were shared between two mapping populations, our results are not obscured by differences in the size of the maps or their coverage of the genome. We found that intraspecific recombination rates were only slightly higher (1.8\%) than interspecific rates, which indicates that the observed genic incompatibilities between Nasonia species do not severely increase or decrease recombination

We found one major incongruence in the marker order between the intraspecific $N$. vitripennis and the interspecific $N$. vitripennis $\times N$. giraulti map. Although we cannot rule out a genomic inversion in one of the two species, we think that this finding likely reflects a mapping error because we do not find such a difference 
in the marker order in any of the other crosses. Thus, when comparing the recombination frequencies between different interspecific crosses with either the same or with a different cytoplasm (Figure 4), we found no significant difference. We had not been able to include markers on chromosome 5 in the interspecific map comparisons. However, our comparison of the intraspecific $N$. vitripennis map with the interspecific $N$. vitripennis $\times N$. giraulti map did include four marker intervals on this chromosome. Chromosome 5, together with chromosome 1 , showed the largest deviation in recombination frequencies between mapping populations. As mapping populations of embryonic and adult $\mathrm{F}_{2}$ hybrids did not differ significantly in their recombination frequencies, we conclude that interspecific crosses with adult individuals do not greatly impair mapping studies in Nasonia. However, we nonetheless advice caution when interpreting differences in recombination frequencies on chromosomes 1 and 5 between mapping populations.

Future studies that address the comparative genomics of closely related species will provide important additional data on the speed and mode of genome evolution, and the Nasonia clade may well have an important function (Werren et al., 2010). With such information at hand, we may gain further insights of the extent to which phylogenetic distance maps to major genomic differences and of the forces that drive the evolution of genomic changes (Lynch, 2007). Comparison of recombination frequencies and the degree of synteny between related species allows us to infer the function of chromosomal rearrangements in species divergence (Ortiz-Barrientos et al., 2002). Recombination frequencies in interspecific hybrids have been well studied in plants in the context of polyploidization and selective breeding of cultured plant species. These studies revealed major alterations of meiotic pairing leading to distortions in recombination frequencies (Zhang et al., 1999). For example, Llopart et al. (2005) found that the degree of introgression between Drosophila yakuba and D. santomea depends on the region in the genome and the associated recombination rate. Recombination between divergent genomes in hybrids may be selectively disadvantageous, because it often leads to hybrid dysgenesis, with a proportionally large effect of the sex chromosomes (Coyne and Orr, 2004). However, beneficial effects have been reported too (for example Edmands, 2008). Nasonia wasps are haplodiploid and do not have sex chromosomes, yet interspecific crosses do result in increased mortality of $\mathrm{F}_{2}$ hybrid males (Breeuwer and Werren, 1995; Gadau et al., 1999; Bordenstein and Werren, 1999; Niehuis et al., 2008; Koevoets and Beukeboom, 2009; Koevoets, unpublished). We found no evidence for major genome rearrangements between the three Nasonia species. However, our genome coverage was only $70 \%$ (intraspecific $N$. vitripennis versus interspecific $N$. vitripennis $\times N$. giraulti map) and $45 \%$ (interspecific $N$. vitripennis $\times N$. giraulti versus $N$. vitripennis $\times N$. longicornis map), respectively, and chromosome 5 was not included in the latter comparison. Interestingly, both Niehuis et al. (2008) and Koevoets (unpublished) have identified chromosome 5 as being involved in male $F_{2}$ hybrid male breakdown. Hence, it remains to be seen to what extent hybrid breakdown in Nasonia is due to detrimental epistatic interactions between genes or due to chromosomal rearrangements.

\section{Conflict of interest}

The authors declare no conflict of interest.

\section{Acknowledgements}

LWB, BAP, and TK acknowledge the Netherlands Organization for Scientific Research NWO/ALW (grants 863.08.008, 816.01.004, and 833.02.003). ON acknowledges the Alexander von Humboldt Foundation for a Feodor Lynen Research Fellowship for Postdoctoral Researchers. BAP and DMS were supported by the National Environment Research Council. JG was supported by a grant from the National Institutes of Health (NIH 1R21 RR024199-01). We are indebted to two anonymous reviewers for valuable comments on an earlier version of the paper.

\section{References}

Bordenstein SR, Werren JH (1999). Effects of A and B Wolbachia and host genotype on interspecies cytoplasmic incompatibility between two Nasonia species. Genetics 148: 1833-1844.

Breeuwer JAJ, Werren JH (1995). Hybrid breakdown between two haplodiploid species: the role of nuclear and cytoplasmic genes. Evolution 49: 705-717.

Burton-Chellew MN, Koevoets T, Grillenberger BK, Sykes EM, Underwood SL, Bijlsma K et al. (2008). Facultative sex ratio adjustment in natural populations of wasps: cues of local mate competition and the precision of adaptation. Am Nat 172: 393-404.

Campbell BC, Steffen-Campbell JD, Werren JH (1993). Phylogeny of the Nasonia species complex (Hymenoptera: Pteromalidae) inferred from an rDNA internal transcribed spacer (ITS2). Insect Mol Biol 2: 225-237.

Chen D, Berger J, Fellner M, Suzuki T (2009). FLYSNPdb: a high-density SNP database of Drosophila melanogaster. Nucleic Acids Res 37: D567-D570.

Coyne J, Orr A (2004). Speciation. Sinnauer Associates: Sunderland, MA.

Darling DC, Werren JH (1990). Biosystematics of Nasonia (Hymenoptera: Pteromalidae): two new species reared from birds' nests in North America. Ann Entomol Soc Am 83: 352-370.

Edmands S (2008). Recombination in interpopulation hybrids of the copepod Tigriopus californicus: release of beneficial variation despite hybrid breakdown. J Hered 99: 316-318.

Fulton RE, Salasek ML, Duteau MN, Black WC (2001). SSC analysis of cDNA markers provides a dense linkage map of the Aedes aegypti genome. Genetics 158: 715-726.

Gadau J, Page RE, Werren JH (1999). Mapping hybrid incompatibility loci in Nasonia. Genetics 153: 1731-1741.

Gadau J, Niehuis O, Peire A, Werren JH, Baudry E, Beukeboom LW (2008). The jewel wasp-Nasonia. In: Hunter W, Kole C (eds). Genome Mapping and Genomics in Animals. Springer Verlag: Berlin Heidelberg. pp 27-41.

Gokhman VE, Westendorf M (2000). The chromosomes of three species of the Nasonia complex (Hymenoptera, Pteromalidae). Beitr Entomol Suppl 50: 193-198.

Hunter W, Kole C (2008). Genome Mapping and Genomics in Animals. Springer Verlag: Berlin Heidelberg.

Koevoets T, Beukeboom LW (2009). Genetics of postzygotic isolation and Haldane's rule in haplodiploids. Heredity 102: 16-23.

Kosambi DD (1944). The estimation of map distances from recombination values. Ann Eugen 12: 172-175.

Kulathinal RJ, Bennett SM, Fitzpatrick CL, Noor MAF (2008). Fine-scale mapping of recombination rate in Drosophila 
refines its correlation to diversity and divergence. Proc Natl Acad Sci USA 105: 10051-10056.

Llopart A, Lachaise D, Coyne JA (2005). Multilocus analysis of introgression between two sympatric sister species of Drosophila: Drosophila yakuba and D santomea. Genetics 171: 197-210.

Lorenzen MD, Doyungan Z, Savard J, Snow K, Crumly LR, Shippy TD et al. (2005). Genetic linkage maps of the red flour beetle, Tribolium castaneum, based on bacterial artificial chromosomes and expressed sequence tags. Genetics 170: 741-747.

Lynch M (2007). The frailty of adaptive hypotheses for the origins of organismal complexity. Proc Nat Acad Sci USA 104: 8597-8604.

Mester DI, Ronin YI, Hu Y, Peng J, Nevo E, Korol AB (2003a). Efficient multipoint mapping: making use of dominant repulsion-phase markers. Theor Appl Genet 107: 1102-1112.

Mester DI, Ronin YI, Minkov D, Nevo E, Korol AB (2003b). Constructing large-scale genetic maps using an evolutionary strategy algorithm. Genetics 165: 2269-2282.

Mester DI, Ronin YI, Nevo E, Korol AB (2004). Fast and high precision algorithms for optimization in large-scale genomic problems. Comput Biol Chem 28: 281-290.

Myers S, Bottolo L, Frreeman C, McVean G, Donnelly P (2005). A fine-scale map of recombination rates and hotspots across the human genome. Science 310: 321-324.

Niehuis O, Judson AK, Gadau J (2008). Cytonuclear genic incompatibilities cause increased mortality in male $F_{2}$ hybrids of Nasonia giraulti and $N$ vitripennis. Genetics 178: 413-426.

Niehuis O, Gibson JD, Rosenberg MS, Pannebakker BA, Koevoets T, Judson AK et al. (2010). Recombination and its impact on the genome of the haplodiploid parasitoid wasp Nasonia. PLoS ONE (doi:10.1371/journal.pone.0008597).

Ortiz-Barrientos D, Reiland J, Hey J, Noor MAF (2002). Recombination and the divergence of hybridizing species. Genetica 116: 167-178.

Pannebakker BA, Halligan DL, Reynolds KT, Ballantyne GA, Shuker DM, Barton NH et al. (2008). Effects of spontaneous mutation accumulation on sex ratio traits in a parasitoid wasp. Evolution 62: 1921-1935.

Pannebakker BA, Niehuis O, Hedley A, Gadau J, Shuker DM (2010). The distribution and evolution of microsatellites in the Nasonia parasitoid wasp genome. Insect Mol Biol 19: 91-98.

Petes TD (2001). Meiotic recombination hot spots and cold spots. Nat Rev Genet 2: 360-369.

Pietsch C, Rütten K, Gadau J (2004). Eleven microsatellite markers in Nasonia, Ashmead 1904 (Hymenoptera; Pteromalidae). Mol Ecol Notes 4: 43-45.

Raychoudhury R, Desjardins CA, Buellesbach J, Loehlin DW, Grillenberger BK, Beukeboom LW et al. (2010). Behavioural and genetic characteristics of a new species of Nasonia. Heredity (doi:10.1038/hdy.2009.147).
Rütten KB, Pietsch C, Olek K, Neusser M, Beukeboom LW, Gadau J (2004). Chromosomal anchoring of linkage groups and identification of wing size QTL using markers and FISH probes derived from microdissected chromosomes in Nasonia (Pteromalidae: Hymenoptera). Cytogenet Genome Res 105: 126-133.

Saul GB, Kayhart M (1956). Mutants and linkage in Mormoniella. Genetics 41: 930-937.

Saul GB (1993). Gene map of the parasitic wasps Nasonia vitripennis (= Mormoniella vitripennis) $(2 \mathrm{~N}=10)$. In: $\mathrm{O}^{\prime}$ Brien SJ (ed). Genetic Maps. Locus Maps of Complex Genomes. Cold Spring Harbor Laboratory Press: Cold Spring Harbor, NY. pp 3: 193-198. .201.

Solignac M, Mougel F, Vautrin D, Monnerot M, Cornuet J-M (2007). A third-generation microsatellite-based linkage map of the honeybee, Apis mellifera, and its comparison with the sequence-based physical map. Genome Biol 8: R66.

Tan YD, Wan C, Zhu Y, Lu C, Xiang Z, Deng HW (2001). An amplified fragment length polymorphism map of the silkworm. Genetics 157: 1277-1284.

Trickett AJ, Butlin RK (1994). Recombination suppressors and the evolution of new species. Heredity 73: 339-345.

Voorrips RE, Jogerius MC, Kanne HJ (1997). Mapping of two genes for resistance to clubroot (Plasmodiphora brassicas) in a population of doubled haploid lines of Brassica oleracea by means of RFLP and AFLP markers. Theor Appl Genet 94: 75-82.

Werren JH, Richards S, Desjardins CA, Niehuis O, Gadau J, Colbourne JK et al. (2010). Functional and evolutionary insights from the genomes of three parasitoid Nasonia species. Science 327: 343.

Weston RF, Qureshi I, Werren JH (1999). Genetics of wing size difference between two Nasonia species. J Evol Biol 12: 586-595.

Whiting AR (1967). The biology of the parasitic wasp Mormoniella vitripennis [ = Nasonia brevicornis] (Walker). $Q$ Rev Biol 42: 333-406.

Wilfert L, Gadau J, Schmid-Hempel P (2007). Variation in genomic recombination rates among animal taxa and the case of social insects. Heredity 98: 189-197.

Yamamoto K, Narukawa J, Kadono-Okuda K, Nohata J, Sasanuma M, Suetsugu Y et al. (2006). Construction of a single nucleotide polymorphism linkage map for the silkworm, Bombyx mori, based on artificial chromosome end sequences. Genetics 173: 151-161.

Zhang L, Pickering R, Murray B (1999). Direct measurement of recombination frequency in interspecific hybrids between Hordeum vulgare and $H$ bulbosum using genomic in-situ hybridization. Heredity 83: 304-309.

Zhang L, Yang C, Zhang Y, Li L, Zhang X, Zhang Q et al. (2007). A genetic linkage map of Pacific white shrimp (Litopenaeus vannamei): sex linked microsatellite markers and high recombination rates. Genetica 31: 37-49.

Supplementary Information accompanies the paper on Heredity website (http://www.nature.com/hdy) 